$$
\text { Fyson, R (2015) }
$$

Building an Evidence Base for Adult Safeguarding? Problems with the Reliability and Validity of Adult Safeguarding Databases

British Journal of Social Work: 45 (3) 932-948

doi: $10.1093 /$ bjsw/bct163

http://bjsw.oxfordjournals.org/content/45/3/932 


\title{
Building an evidence base for adult safeguarding? Problems with the reliability and validity of adult safeguarding databases
}

\begin{abstract}
This article considers current adult safeguarding policy guidelines for England, which require local authorities to collect adult safeguarding data for the purposes of research and service development. It then moves on to report some of the findings from an evaluation of adult safeguarding in one English local authority, focussing on how the adult safeguarding database was populated from case records and how the resultant data was utilised. It found that, although the annual number of adult safeguarding alerts more than tripled between 2002 and 2008, this clear evidence of an increase in workload had not resulted in increased resource allocation. The evaluation further noted that only half of the designated 'adult safeguarding managers' who were interviewed were able to correctly define the meanings of the recommended terms under which adult safeguarding outcomes are recorded, i.e. 'substantiated', 'not substantiated' and 'not determined'. Changes to the terminology used to record the outcomes of safeguarding investigations are proposed as one of a number of measures to enable the creation of valid and reliable information upon which to base future practice developments, including allocation of resources.
\end{abstract}

Key words: adult safeguarding; adult protection; information \& computer technology; safeguarding database; evidence based practice; 


\section{Introduction}

As a field of social work practice which has largely developed during the 'information age', adult safeguarding has, from the outset, paid careful attention to the need not only for effective practice but also for effective case recording and statistical monitoring of practice outcomes. In this context, social workers are required not simply to maintain accurate case records but to do so in such a way that this information can also be used as monitoring data. This practice may be construed as part of the increasingly managerialist turn in social work (Lymbery, 2001 \& 2004; Healy \& Meagher, 2004) or may be understood as deriving from the increasing emphasis on evidence-based practice (Webb, 2001; Sheldon, 2001), both of which broadly coincided with a burgeoning awareness of the abuse of some groups of 'vulnerable' adults (Fitzgerald, 2008).

In theory, better monitoring data should not only afford opportunities to enhance understandings of risk and provide evidence of 'what works' in adult safeguarding, but should also inform management decisions such as resource allocation. However, despite the fact that national guidelines on data collection have been published, and the collection and publication of monitoring data on adult safeguarding is now commonplace across England, concerns remain about the "nature and quality of the data collected and reported" (Manthorpe et al, 2011, p.i). This paper will provide evidence that monitoring data which is currently being used to develop 'evidence-based' practice may be both unreliable due to regular changes in the categories used within databases, and (sometimes) invalid due to problems in the relationship between case records and their use as monitoring data. Furthermore, it will suggest that, even when monitoring data is reliable and valid, local authorities may not - in the present economic climate - be willing or able to use this information to allocate increased resources to adult safeguarding.

\section{Background}

There is evidence that some local authorities were "collating adult protection and monitoring data in a form that enabled basic comparisons as early as 1998" (Cambridge, 2009, p.2). These practices were put on a more formal footing following the publication of the first (and, to date, only) national policy guidelines on adult safeguarding, No Secrets (Department of Health and Home Office, 2000), which included the following specific guidance:

Whenever a complaint or allegation of abuse is made all agencies should keep clear and accurate records and each agency should identify procedures for incorporating, on receipt of a complaint or allegation, all relevant agency and service user records into a file to record all action taken. [... ... ] Records should 
be kept in such a way that they create statistical information as a by-product. (Department of Health \& Home Office, 2000, p.30, emphasis added)

This guidance implies the need for case records to be maintained in a consistent manner, so that the 'by-product' of 'statistical information' is made possible. It further implies that this information will be used purposefully, presumably for quantitative analysis of factors thought relevant to the detection or prevention of abuse and therefore the commission of suitable services.

However, despite the collection of statistical information by some authorities, and its use by local researchers to contribute to an emerging evidence base (see, for example, Brown \& Stein, 1998 \& 2000), not all authorities were quick to adopt this aspect of No Secrets. One early report on the implementation of No Secrets, based on documentary analysis of local authority codes of practice, commented that "robust information systems are a key element in strategic and operational planning" (Sumner, 2002, p.4), but also noted how:

The translation of initial consultation activity and the development of data collection and analysis (currently at an early stage) will hopefully, over time, lead to an increase in its role in informing service development priorities, presently only referred to in $9 \%$ of the codes. (ibid, p.5)

Partly in response to these early concerns about the limited engagement of many local authorities with record keeping for the purposes of audit and evidenced-based practice, the Department of Health commissioned Action on Elder Abuse to examine this aspect of adult safeguarding. The resulting report (Action on Elder Abuse, 2006) made the case for monitoring data to be collected consistently by all local authorities and included specific guidelines on how the outcomes of adult safeguarding investigations should be recorded (see Box 1: recommended terms for recording outcomes). The call for systematic collection of monitoring data was also backed by ADASS (Association of Directors of Adult Social Services), whose National Framework of Standards for good practice and outcomes in adult protection work called on local authorities to ensure that "There are effective arrangements for the monitoring of 'Safeguarding Adults' work by partner agencies and for the collation of data" (ADASS, 2005, p.9). 


\section{Box 1: Action on Elder Abuse (2006) Adult Protection Data Collection and Reporting Requirements: Conclusions and recommendations from a two year study into Adult Protection recording systems in England, funded by the Department of Health London: Action on Elder Abuse. www.elderabuse.org.uk}

Page 22: 5.1 .13

Case conclusions should be based on four categories. The burden of proof should be consistent with the standard applied to the Protection of Vulnerable Adults (POVA) List which is 'on the balance of probabilities'.

The categories are:

Substantiated - All of the allegations of abuse are substantiated on the balance of probabilities.

Partly Substantiated - This would apply to case where it has been possible to substantiate some but not all of the allegations made on the balance of probabilities. For example 'it was possible to substantiate the physical abuse but it was not possible to substantiate the allegation of financial abuse'.

Not Substantiated - It is not possible to substantiate on the balance of probabilities any of the allegations of abuse made.

Not Determined/Inconclusive - This would apply to cases where it is not possible to record an outcome against any of the other categories.

Despite the slow start identified by Sumner (2002), it is evident that many - perhaps most - local authorities are now collecting adult safeguarding monitoring data broadly in accordance with No Secrets guidelines. Within this, it is impossible to say precisely how many are using the Action on Elder Abuse recommended terms as their standard way of recording case outcomes. It is, however, certain that many have done so: see, for example, annual reports on adult safeguarding from the London Borough of Sutton, Middlesbrough Council or Staffordshire County Council (all 2011). These reports use monitoring data collected by Local Safeguarding Adults Boards and they typically contain - amongst other things - information about: the number of referrals received; service user group of alleged victim; nature of abuse identified; where abuse took place; relationship of victim to alleged perpetrator, etc. Many also contain statistics detailing the outcomes of referrals, including changes in service provision for the alleged victim and whether criminal proceedings have been initiated against the alleged perpetrator. Local monitoring data has also been used as the basis for academic research, which seeks to inform wider debates and developments within adult safeguarding (see, for example Cambridge et al, 2010 \& 2011; Thacker, 2011; Beadle-Brown et al, 2008). At the same time, local authority adult safeguarding managers have continued to call for 
further "analysis of safeguarding data to understand and mitigate risk factors" (Reece, 2010, p.33).

\section{About the present study}

The findings reported in this paper emerged during an evaluation of adult safeguarding in one English local authority. The findings were unexpected, in that the study inadvertently uncovered serious issues in relation to the accuracy of case outcome data held in the adult safeguarding database. In order to explain this, it is necessary to provide a brief overview of the evaluation process as a whole before moving on to explore pertinent aspects of case recording and its relationship to monitoring data. The evaluation of adult safeguarding services was originally commissioned due to concerns about a perceived increase in the number of adult safeguarding investigations resulting in 'inconclusive' outcomes. Managers were keen not only to establish whether this perception was borne out by local adult safeguarding data, but also to identify factors which contributed to inconclusive outcomes. The evaluation consisted of three phases: statistical analysis of information from the adult safeguarding database; analysis of a sample of recent safeguarding adult assessment outcomes, in order to identify factors which contributed to securing a definitive outcome; and interviews with Safeguarding Managers from Adult Social Care and Health teams. Ethical approval was obtained from the University of Nottingham.

The adult safeguarding database made available to this study included all data collected by the authority between November 2001 and January 2009, so complete data was available for years 2002 - 2008. Statistical analysis of this data aimed to provide an exploration of the links between different vulnerable adult groups; different types of abuse; different circumstances of abuse, etc and the outcomes of safeguarding alerts. For reasons which will become apparent, most of this statistical analysis is not presented.

Findings from the second phase of the evaluation are reported elsewhere (Author, 2012), but - in summary - there was evidence to suggest that a range of factors impact upon the likelihood of achieving conclusive outcomes following an adult safeguarding investigation. These included not only victim characteristics, but also inter-agency cooperation; social workers' pre-existing knowledge of the alleged victim; and whether or not a formal safeguarding planning meeting was convened.

For the final phase of the evaluation, interviews were undertaken with 14 designated 'Safeguarding Managers'; this meant that the interviewee was responsible for ensuring that all adult safeguarding investigations undertaken by members of the team which 
they managed were completed to a satisfactory standard, within expected timeframes, and recorded on the safeguarding database. Safeguarding Managers were drawn from across the full range of adult social care specialist teams including: physical disabilities and vulnerable adults; adult care management; intermediate care; community mental health; community learning disability; and older person's teams. All Adult Social Care and Health team managers were invited to interview; actual participants comprised an opportunity sample of those able to be interviewed within a two-week timeframe. The eventual sample nevertheless comprised over half of all designated Safeguarding Managers and included managers from every type of adult social care fieldwork team and from across all geographic areas of the local authority.

The interviews were semi-structured and each followed the same pattern of questions, starting by asking about the interviewees' personal experience of safeguarding training and safeguarding work, moving on to discuss current safeguarding processes - including how cases were recorded on computer systems; interagency working; decisions about whether abuse allegations were substantiated; inputting of outcomes data onto the adult safeguarding database for monitoring purposes- and concluding with a discussion of the impact of safeguarding work on social workers. The use of semi-structured interviews is a recognised approach within qualitative research; it enables data to be collected in a rigorous and methodical manner whilst allowing the interviewer to modify the sequence or wording of questions where necessary (Becker et al, 2012). Each interview lasted for between 30 and 50 minutes and all were audio-recorded with the informed consent of participants.

Since the work was commissioned as a service evaluation rather than as an academic study, the available budget did not run to full transcription of interviews. Instead, the author repeatedly re-played the interview recordings, noting key themes, similarities and differences between interviews in an iterative process of thematic content analysis. This, together with extensive contemporaneous post-interview notes, enabled a systematic analysis of interview data. Those parts of the interviews which explored practices of recording (case records) and monitoring (the collection of data for audit purposes) are the focus of this paper: sections of interview data relating to these themes were transcribed in full to provide illustrative quotes.

\section{Reliability of monitoring data held in the Adult Safeguarding database}

The database provided clear evidence of a significant increase over time in the number of adult safeguarding alerts received by the authority. During the seven years for which full annual data were available ( $1^{\text {st }}$ January $2002-31^{\text {st }}$ December 2008 ), the number of 
recorded adult safeguarding alerts increased more than threefold, from 329 alerts in 2002 to 1,105 alerts in 2008.

However, although the monitoring data provided a clear picture of numbers of safeguarding alerts, there were difficulties in using much of the other information in the safeguarding database. These difficulties arose in part because the pro forma which social workers completed, which was then copied into an Excel database, was designed to meet the needs of professionals undertaking safeguarding adult assessments and recording case files rather than the needs of audit. These difficulties were compounded by problems of reliability - due to missing data, ambiguous data and regular changes in terminology over time, with resultant unquantifiable changes in recording practice. Whilst only very limited statistical analyses of the data are presented in this paper, the problems with monitoring data are important because of the challenge they pose to the expectation that adult safeguarding databases can be used to help build a reliable evidence-base for practice.

Missing data included a small number of cases which had to be excluded because no referral date was recorded (44 out of 4086 cases, or $1.07 \%$ ). More significantly, records did not include the gender of either alleged victims or alleged perpetrators until 2004, rendering gender analysis impossible.

Ambiguity within the data took a number of forms, but mostly arose because professionals completing the records used a range of different terms which may or may not have been intended to refer to the same category of person. Sometimes it was possible to have a reasonable degree of confidence when interpreting categories: for example, in relation to 'who made the safeguarding alert?' the categories of 'alleged victim' and 'vulnerable adult themselves' were collapsed to create one category for 'alleged victim'. At other times the confidence with which codes could be re-categorised was lesser: for example, in relation to 'who was the alleged abuser?' the categories 'family members' and 'partners' and 'main family carers' were collapsed together, although it could not be certain whether each of these implied that victim and alleged perpetrator shared a home. Finally, there were times when information had been recorded in a manner which may have been helpful within case records, but was unusable for monitoring purposes: for example, the alleged perpetrator was sometimes recorded as a named individual (e.g. 'John Smith') rather than in terms of their relationship to the alleged victim (e.g. 'spouse' or 'care staff').

During the period over which monitoring data had been collected, many changes had taken place in terminology - not least the switch from 'adult protection' to 'adult safeguarding'. However, the terminology changes which had the most serious implications for statistical analysis (in light of the original remit, which was to investigate 
inconclusive outcomes) were changes to how outcomes were recorded. At different points in time, both 'disproven' and 'not substantiated' were used to indicate what appeared to be the same outcome i.e. that alleged abuse was not believed to have taken place. Likewise, 'proven' and 'substantiated' were variously used to indicate that the alleged abuse had occurred. Where outcomes were less clear-cut, the terms 'inconclusive', 'not determined' and 'possible' were all used. For purposes of analysis, 'disproven' and 'not substantiated' were put together into the category of 'not substantiated', whilst 'proven' and 'substantiated' were put together into the category of 'substantiated'. Similarly, 'inconclusive' and 'not determined' were put together as 'not determined. But 'possible' was retained as a separate category owing to the frequency with which it was used over a number of years.

Figure 1: Recorded outcomes, by year, $2002-2008$

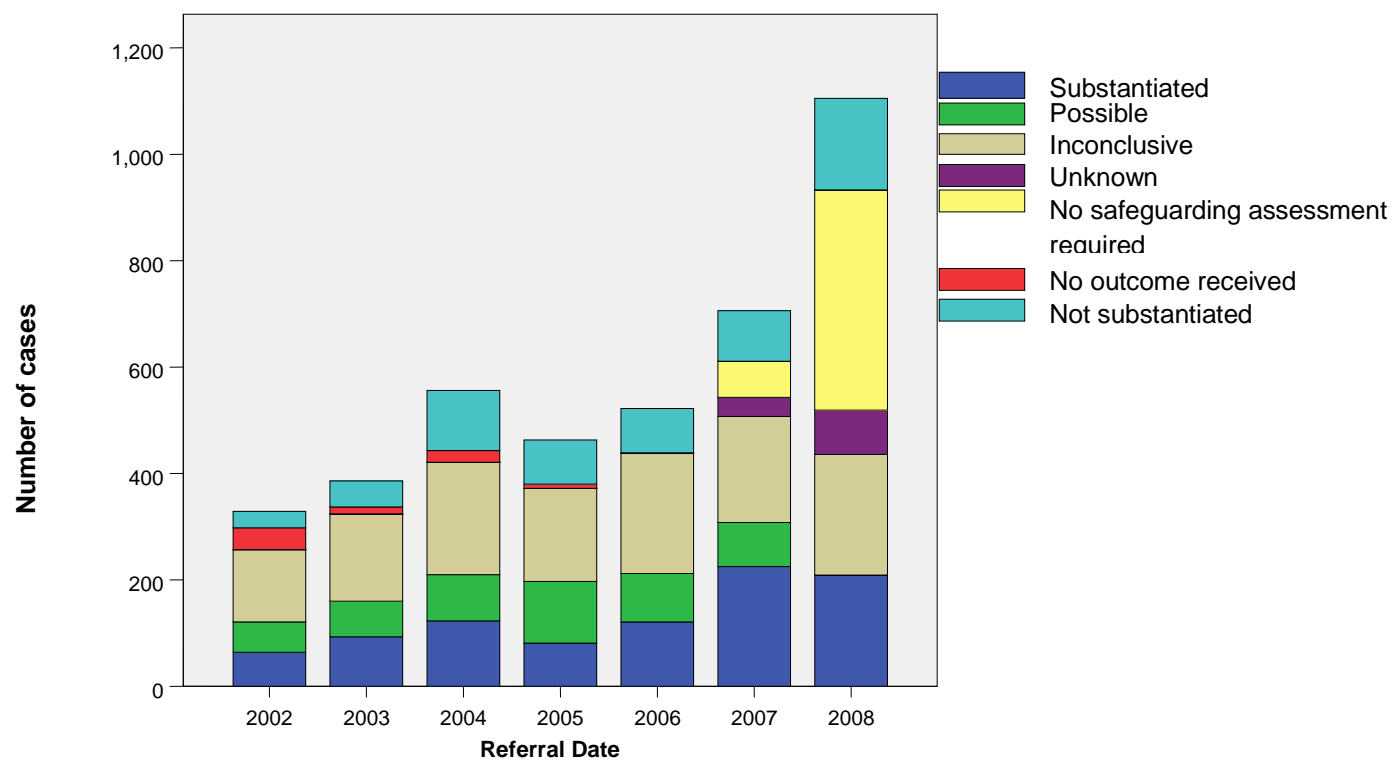

The impact of these changes in terminology can, in part, be seen in Figure 1: Recorded outcomes, by year, 2002 - 2008. Notwithstanding any questions regarding the validity of coding categories which had been applied post hoc, analysis of available monitoring data showed that, despite the number of safeguarding alerts increasing substantially over time, the number of cases resulting in an inconclusive outcome remained relatively stable. This meant that the proportion of alerts which resulted in an 'inconclusive' outcome was, in fact, falling. What was also striking, however, was how uses of certain categories had changed over time. For example, the 'possible' category stopped appearing in 2008, having previously accounted for 50-100 cases each year; the 
category 'no outcome received' was present between 2002-2005, but the new categories of 'unknown' and 'no safeguarding assessment required' were only introduced in 2007. The category of 'no safeguarding assessment required' is particularly interesting, in that it coincided with both the switch from adult protection to adult safeguarding and a dramatic increase in the overall number of alerts being received. There was speculation amongst interviewees that the increase in alerts had a causal relationship with introduction of the notion of 'safeguarding', as this had precipitated more active awareness-raising amongst service provider organisations. This appeared in turn to have resulted in greater numbers of alerts being made, a significant proportion of which were inappropriate (i.e. did not require a full safeguarding assessment).

The changing nomenclature used within the safeguarding database raises questions about the validity of any statistical analysis of the resultant dataset. It will have inevitably introduced a substantial element of unreliability as it is impossible to quantify how practitioners will have interpreted the different categorisations used over time.

\section{The use of monitoring data to manage resources}

Despite concerns about the reliability and consistency of information held within the adult safeguarding database, the one fact which was clearly evidenced was a substantial increase in the number of safeguarding alerts received by adult social care teams $(300 \%$ increase in seven years). Interviewees were keenly aware from personal experience that the volume of safeguarding-related work had increased markedly. They were concerned about the proportion of their working hours which safeguarding now consumed and did not believe that sufficient resources (in terms of additional person-hours) had been forthcoming to ameliorate the impact of increased safeguarding alerts on their overall workload.

It's probably more than a third of my working time. I wouldn't think it was a half, but it has grown. In the time I have been here I would say that safeguardings were a regular minority of the work that I dealt with; now they're becoming almost a daily, if not two-daily, occurrence. I'm not saying they go into full safeguardings, but the referrals coming through are almost daily.

I certainly don't think we're resourced effectively and efficiently enough to cope with the increasing numbers that are going to come through.

They also spoke of how difficult it could be to ensure safeguarding assessment deadlines were met without compromising the quality and quantity of their 'bread and butter' (i.e. 
assessment and care management) work. In many cases, it was recognised that these deadlines could not always be met, especially when a large number of safeguarding alerts were received within a short period of time.

If this last year is anything to go by the numbers are going to continue to grow, in which case we won't cope with the numbers that are coming through. Or, if we are having to cope with those, we won't be doing the bread and butter work. Our daily job will suffer as a result of us having to continually meet the demands and the timescales on the safeguardings that are coming in. So it's not so much for me about the process, it's about how we resource what we've got to do.

Worryingly, a number of interviewees indicated that these pressures were resulting in some staff adopting a negative attitude towards safeguarding work:

Workers don't like it at all. It's the worst thing, if you're on duty, if you get a safeguarding case in.

I think they feel that this work can be very satisfying, but I also think that they feel that it gets in the way sometimes, because they're not able to get on with the day-to-day stuff as well. And I think that leaves them feeling very stressed. I don't think that's the safeguardings' fault per se, I think the nature of the work, the time it takes and the volume of it makes them feel like that.

In effect, it appeared that although frontline staff were struggling with increasing safeguarding referrals, evidence of a substantial increase in demand for adult safeguarding had not resulted in any increase in resources.

\section{Care records or monitoring data? Emergent questions of reliability and validity}

The computerised recording system which social workers used was devised as a means to maintain accurate cases records, with monitoring data produced (in line with national guidelines) as a by-product of the case recording process. The practice was for monitoring data to be imported into the safeguarding database from existing electronic case records. Many interviewees were critical of the computerised systems used to record details of safeguarding assessments, finding the 'paperwork' both unhelpful and repetitive:

The paperwork that we use doesn't flow very easily, either [...] It doesn't follow on very well. So, yeah, I think if we had better paperwork it would be a lot easier. 
It's not the easiest paperwork to work with and a lot of it is very repetitive. I would generally go out and do my investigation and then fit it into the paperwork [...] slot it into the right boxes.

Even those who were less critical acknowledged that they did not use all parts of the system in the prescribed manner:

I don't tend to go into the strategy document. I find that really, it's very repetitive and there's a lot there that needs to be included in the investigating officers report. So I, personally I tend to get it all documented in the investigating officers report, with times, dates and discussions with whom, etc. So I don't tend to use that part of it. I do follow the investigating officer's format and I do follow the outcome form format. I don't deviate from that, I use that as the template for how I set out the investigation and the outcome. And also with regard to reviews as well I do use the documentation as it's laid out

At the end of each safeguarding assessment, there was a requirement to formally record an outcome of 'substantiated', 'not substantiated' or 'not proven' (cf. Action on Elder Abuse, 2006). Opinions amongst interviewees differed as to whether the final decision regarding the recorded outcome of a safeguarding assessment was a straightforward matter, following logically from the information gathered, or a challenging moment in which professional judgement came under intense pressure. Part of the variation was undoubtedly due to the different types of cases which interviewees had typically dealt with. For example, where carers reported their own abusive behaviour, the purpose of the investigation was no longer about establishing whether abuse had occurred, but was aimed at identifying how support could be restructured or enhanced to ensure that such events were not repeated. Other situations were more complex, with safeguarding investigators having to decide between claim and counter-claim on the basis of limited evidence. Many interviewees reported that alerts about alleged abuse in a service users' home, with no witnesses other than the alleged victim and alleged abuser, were almost impossible to resolve definitively.

Some safeguardings are, some you are never going to come to an outcome that will prove it definitely one way or t'other. There are a lot where the information that's received is very ambiguous and if you can't get it clarified, if you can't get somebody to corroborate it, if it's one person's word against another, it's very, very difficult to come out with an outcome that's satisfactory. And when you're working with people that's always going to be the case, isn't it?

Given the cognitive deficits and communication difficulties of many people subject to adult safeguarding investigations, the frequent reliance on hearsay in the absence of 
physical evidence, and the lack of consensus between professionals about the process by which safeguarding decisions should be made, it is inevitable that recorded outcomes are subject to some degree of human variation. In other words, it is unsafe to assume that the outcome recorded in any given case would have been the same if a different professional had taken the lead on investigating and recording. But whilst some variation in professional judgement is probable, the adherence to a shared set of social work values can be expected to result in outcomes which lie within an acceptable margin of difference. This study provided no evidence to contradict the assumption that professional decisions lay within an acceptable range. However, it did reveal serious concerns about the validity the outcomes recorded for monitoring purposes.

Because the author had found the terminology used for recording case outcomes somewhat opaque and because of evidence of changes in the use of terminology over time, all interviewees were asked to define - in so far as they understood them - the terms used to describe and record the outcomes of their safeguarding assessments.

Every interviewee was able to give a definition of 'substantiated' which broadly coincided with the definition adopted by the local authority, as set out by Action on Elder Abuse (2006) - i.e. that, in so far as could be established and on the balance of probabilities, abuse had taken place. Moreover, where this outcome was recorded, interviewees expressed a high degree of confidence that the outcome was a true reflection of events.

If something's substantiated then I think there's overwhelming evidence to suggest it actually happened.

I'm confident. I wouldn't say I'm $100 \%$ every time, but I'm confident. I wouldn't tick it if I wasn't.

Nevertheless, concern arose for some over whether choosing this outcome was an indication simply that the alleged victim had indeed suffered abuse or a statement that the abuse had been perpetrated by the alleged abuser. It was suggested that this may be a reason why, when a specific individual is named as the alleged abuser, some social workers may be reluctant to substantiate abuse, even when they believe abuse to have occurred - for example in a care home where there is clear evidence of non-accidental physical injury, but it is not possible to say which member of staff was responsible.

The wording we have at the end of a safeguarding assessment is very woolly and not very clear. And I think people get very worried about ticking a box that says this has been substantiated. Because 9 times out of 10 we haven't proved that that abuse has gone on. We know that it has, but we can't say for certain that this person has done it. This is the person we suspect. And we've gone as far as we can with the investigation. And we've made sure the person is not at risk any 
more. But people don't want to tick substantiated because it makes it sound like we're saying 'this person has done this; this person has financially abused this person or this person has hit this person or whatever.' [... ...] So people get very funny about ticking substantiated. But we know that that has happened. The person has a bruise on their face or a bruise on their arm or whatever. But because we don't always know who's done it, people don't want to tick it.

By contrast, only half ( 7 out of 14 ) of the interviewees gave an accurate definition of 'not substantiated' - i.e. that, in so far as can be established and on the balance of probabilities, the alleged abuse did not take place. For those who understood the term correctly, this outcome was used with a high degree of confidence, equal to that in cases where the outcome of 'substantiated' was recorded.

Not substantiated is when the investigating officer clearly finds that what was said or what was alleged is not true. It did not happen. And it did not cause the risk, the harm, or whatever. It didn't happen.

However, the other seven interviewees incorrectly believed that 'not substantiated' was the outcome used to record those cases where they were unable to say whether or not abuse occurred.

Not substantiated: It's where, through the investigation, you could identify that it's a high possibility that that allegation is substantiated, but you haven't got any clear evidence that it is.

Not substantiated: Clearly there isn't sufficient evidence to lead us to believe that the allegation ever happened, or it wasn't to the claimed extent, or that we can't substantiate it.

Those interviewees who correctly defined 'not substantiated' were all also able to provide the correct definition of 'not determined' - i.e. that, following a full safeguarding assessment, it remained impossible to say whether or not the alleged abuse had actually taken place. However, those interviewees who had failed to give the correct definition of 'not substantiated' were also unable to provide a correct definition of 'not determined', with some believing it indicated that abuse had not taken place and others recognising that it indicated an uncertain outcome, but unable to say how this differed from their (erroneous) definition of 'not substantiated'.

Not determined: Well, I use that as 'there is nothing'. There is no evidence to suggest whatsoever that that allegation has been substantiated.

Not determined... I think that's more tricky in some respects. I think that's moving... it's like your tariff almost, isn't it, it's down the tariff, not determined. 
Notably, the degree of confidence in cases where the recorded outcome was 'not determined' varied widely between interviewees. This may have been in part because of the different understandings of the terminology. However, even amongst those interviewees who provided full and correct definitions of all terms, there was a range of factors at play when the 'not determined' outcome was used. For some, this was used only in those situations where there was quite simply no way of finding out what had or had not happened - often in cases where the cognitive impairment of an alleged victim was such that they were unable to provide an account of events, or where the alleged victim lived in their own home and the veracity of events was simply one person's word against another. By contrast, others acknowledged that they used this outcome in cases where they were fairly sure that abuse had occurred, but did not have sufficient evidence to prove the case.

I don't think I could say with certainty that I'm neutral, no. No. I think I, I, I would say that in all cases when I tick undetermined or not determined that I just don't have enough to prove that it happened. Not that it didn't happen: I don't have enough to prove that it did happen.

Whilst these findings clearly give rise to a number of further questions about the validity of outcomes recorded on adult safeguarding databases, it is important to stress that they should not be taken as implying anything negative about safeguarding practices. Regardless of their ability to define the outcome categories correctly, interviewees emphasised that - irrespective of an investigation's outcome - the very fact of undertaking a safeguarding assessment could lead to significant change in the support offered to service users. Even where investigations were inconclusive, safeguarding assessments could result in new support packages being put in place; improvements in the standards of care delivered by residential care homes or domiciliary care providers; or family members becoming more aware of the needs of a vulnerable relative and offering additional support.

\section{Discussion and suggestions for improving practice}

The findings from this study give rise to a number of concerns, including: the failure to follow No Secrets recommendations (Department of Health \& Home Office, 2003) regarding the maintenance of adult safeguarding records in such a way that they could be used for both day-to-day practice and research purposes; the apparent confusion amongst Safeguarding Managers about the terminology used to record safeguarding outcomes; and the inability or unwillingness of budget-holders to make more resources 
available for adult safeguarding in response to evidence of a substantial increase in adult safeguarding alerts. Each of these issues will be considered in turn.

Although this local authority was attempting to follow No Secrets guidance on recording adult safeguarding data, it was notable that the pro forma used by social workers to record outcomes following safeguarding assessment was not designed, or completed, with the understanding that it was to be used to collect monitoring data. In this context, the frequent changes in terminology for recording outcomes were likely to have been made without thought for the impact on the value of the information for research or service evaluation purposes.

Evidence from this study would suggest that more attention needs to be paid to both what is recorded and how it is recorded, in order to facilitate the development of a reliable and valid evidence base for improving future practice. In considering recording practices it is vital that adult safeguarding avoids following in the footsteps of child protection and probation, where recording information on elaborate computerised systems has limited the ability of frontline social workers to engage in face-to-face contact with service users (Pemberton, 2010; White et al, 2010; Travis, 2011). It is undoubtedly important, however, that greater attention is now paid to how the outcomes of adult safeguarding alerts are recorded.

Only half of adult safeguarding managers in this study fully understood the terminology used to record case outcomes. This finding alone gives rise to serious questions regarding the validity of the information on the local authority's adult safeguarding database. Whilst it cannot be certain whether these findings would be replicated in other authorities, evidence from publicly-available annual reports from Local Adult Safeguarding Boards (e.g. London Borough of Sutton, 2011; Middlesbrough Council, 2011; Staffordshire County Council, 2011) suggests that the terminology proposed by Action on Elder Abuse (2006) is widely used. And it would seem unlikely that confusion over the terms 'substantiated', 'not substantiated' and 'not determined' are limited to professionals in one geographical location. Notably, some of the authorities which pioneered data collection in adult protection have chosen not to use the recommended terminology, but instead refer to outcomes as 'case confirmed', 'insufficient evidence' and 'case discounted' (Cambridge et al, 2010).

This study further suggests that the value of local authority adult safeguarding databases is likely to be severely compromised by the poor quality of the information they contain. A number of simple changes to recording practices could make a big difference to the validity and reliability of these databases. Firstly, there is an urgent need to adopt more intuitive terminology for denoting the outcomes following adult safeguarding investigations. Neither 'substantiated' or 'determined' are words in common 
usage and, as evidenced in this paper, their use serves to obfuscate rather than illuminate. Secondly, there is a good argument for recording systems to include more 'forced choice'. Whilst the choices made available would need careful piloting, this approach could ensure the accurate recording of key demographic data which is of great value for audit and research purposes: recording the perpetrator as 'John Smith' rather than 'spouse' would no longer be possible. Thirdly, given the finding that some social workers were reluctant to use 'substantiated' because of the implications for the alleged perpetrator, further consideration should be given to what is meant by an 'outcome' and how this should be recorded. In particular, there may be merit in recording whether alleged abuse is believed to have occurred separately from whether it has been possible to identify a perpetrator.

The finding that adult safeguarding alerts had radically increased over recent years is unsurprising, given that this has been widely reported in other studies (Action on Elder Abuse, 2006; Cambridge et al, 2010). Having data available which clearly evidenced social workers' perceptions of a substantial workload increase should have been valuable: the implicit 'rules' of evidence-based practice (i.e. that practices, including practices of resource allocation, should change in response to evidence) would suggest that a hike in the number of safeguarding alerts should lead to more resources. In practice, however, there was no evidence that the increase in alerts had led to any increase in resources. In a context where a $300 \%$ increase in referrals over seven years was not sufficient to trigger additional resources, it is hardly surprising if - working under pressure - frontline staff had little time or incentive to prioritise accurate recording. However, as budgets come under increasing pressure from cuts at national and local levels, and the demographics of an ageing population continue to increase adult safeguarding workloads, there remains an urgent need for reliable data to be collected and used to defend this important area of social work practice.

Whilst this study found no reason to question the quality of adult safeguarding practice, it was evident that information on the adult safeguarding database was of dubious quality and therefore limited value. Furthermore, the one fact which was unequivocally demonstrated - i.e. a rapid increase in the volume of adult safeguarding alerts - had not resulted in any increase in resources. This situation risks the creation of a downward spiral in which overstretched frontline staff do not pay sufficient attention to how they record information about case outcomes and managers may then argue that the available evidence is not sufficiently robust to use as the foundation for evidence-based resource-allocation.

For evidence based practice to flourish, these findings would suggest that greater buy-in from both managers and practitioners is required in order to create a virtuous circle of 
evidence and effect. Current policy relating to the dual use of adult safeguarding records as both case files and monitoring data is sensible, but it would appear that actual practice lags some way behind the desired outcome. Moving forwards, greater consideration needs to be given to the construction and maintenance of databases if they are to achieve an optimal balance between their dual roles of case recording and service monitoring. If this can be achieved, adult safeguarding databases are a potentially invaluable source of information for developing evidence-based practice in identifying and responding to shifting patterns of abuse.

Acknowledgements: Thanks go to Deborah Kitson and to Liz Fox both of whom played important roles in helping to plan and execute the evaluation on which this paper is based.

\section{References}

Action on Elder Abuse (2006) Adult Protection Data Collection and Reporting Requirements: Conclusions and recommendations from a two year study into Adult Protection recording systems in England, funded by the Department of Health London: Action on Elder Abuse

ADASS (2005) Safeguarding Adults: A National Framework of Standards for good practice and outcomes in adult protection work London: ADASS

Beadle-Brown, J; Mansell, J; Cambridge, P; Milne, A \& Whelton, B (2008) Adult protection alerts for people with intellectual disabilities: Patterns of risk. Journal of Intellectual Disability Research, 52 (8-9). p. 788

Cambridge, P (2009) Building on evidence-based practice Journal of Adult Protection 11 (1) $2-5$

Cambridge, P; Beadle-Brown, J; Milne, A; Mansell, J \& Whelton, B (2010) Adult protection: The processes and outcomes of adult protection referrals in two English local authorities Journal of Social Work 11 (3) 247-267

Cambridge, P; Beadle-Brown, J; Milne, A; Mansell, J \& Whelton, B (2011) Patterns of Risk in Adult Protection Referrals for Sexual Abuse and People with Intellectual Disability Journal of Applied Research in Intellectual Disabilities 24 (2) 118-132 
Becker, S; Bryman, A \& Ferguson, H (2012) Understanding Research for Social Policy and Social Work: Themes, Methods and Approaches, $2^{\text {nd }}$ edition. Bristol: Policy Press

Brown, H \& Stein, J (1998) Implementing Adult Protection Policies in Kent and East Sussex Journal of Social Policy 27 (3) 371-396

Brown, H \& Stein, J (2000) Monitoring adult protection referrals in 10 English local authorities Journal of Adult Protection 2 (3) 19-31

Department of Health (2009) Safeguarding Adults: Report on the consultation on the review of 'No Secrets' London: Crown Copyright

Department of Health and Home Office (2000) No Secrets: guidance on developing and implementing multi-agency policies and procedures to protect vulnerable adults from abuse London: Crown Copyright

Healy, K \& Meagher, G (2004) The Reprofessionalization of Social Work: Collaborative Approaches for Achieving Professional Recognition British Journal of Social Work 34 (2) $243-260$

Fitzgerald, G (2008) No Secrets, Safeguarding Adults and Adult Protection in J. Pritchard [ed.] Good Practice in Safeguarding Adults London: Jessica Kingsley

Fyson, R \& Kitson, D (2012) Outcomes following adult safeguarding alerts: a critical analysis of key factors Journal of Adult Protection, 14 (2) 93-103

London Borough of Sutton (2011) Sutton Safeguarding Adults Board Annual Report 2010-2011 https://www.sutton.gov.uk/CHttpHandler.ashx?id=16772\&p=0 (accessed 2.7.12)

Lymbery, M (2001) Social Work at the Crossroads British Journal of Social Work 31 (3)369-384

Lymbery, M. (2004) Managerialism and care management practice with older people, in Lymbery, M \& Butler, S (eds), Social Work Ideals and Practice Realities

Manthorpe, J; Stevens, M; Hussein, S; Heath, H \& Lievesley, N (2011) The abuse, neglect and mistreatment of older people in care homes and hospitals in England: observations on the potential for secondary data analysis Kings College London: Social Care Workforce Research Unit

Manthorpe, J; Tinker, A; McCreadie, C; Biggs, S; Doyle, M; Erens, B \& Hills, A (2008) Number and nuances: the implications for adult protection co-ordinators and committees of the UK national prevalence study of abuse and neglect of older people Journal of Adult Protection 10 (1) 13-23 
Middlesbrough Council (2011) Middlesbrough Safeguarding Vulnerable Adults Committee Annual Activity Report 2010 - 2011

http://www.middlesbrough.gov.uk/CHttpHandler.ashx?id=1083\&p=0 (accessed 2.7.12)

Pemberton, C (2010) Red tape 'undermining' social workers in child protection Community Care, 15th March 2010

Reece, A (2010) Leading the change from adult protection to safeguarding adults: more than just semantics Journal of Adult Protection 12 (3) 30-34

Sheldon, B (2001) The Validity of Evidence-Based Practice in Social Work: A Reply to Stephen Webb British Journal of Social Work 31 (5) 801-809

Staffordshire County Council (2011) The Staffordshire and Stoke-on-Trent Safeguarding Adults Partnership Annual Report 2010-2011 http://www.staffordshirecares.info/Images/Exportfinal.pdf (accessed 2.7.12)

Sumner, K (2002) The Protection of Vulnerable Adults from Abuse: Findings from an analysis of local codes of practice. London: Centre for Policy on Ageing

Thacker, H (2011) Safeguarding adults' referrals in the Eastern region: an investigation into varying referral rates Journal of Adult Protection 11 (3) 137-149

Travis, A (2011) Probation officers spend 75\% of time not dealing with offenders, report finds The Guardian, $27^{\text {th }}$ July 2011

Webb, S (2001) Some considerations on the validity of evidence-based practice in social work British Journal of Social Work 31 (1) 57-79

White, S; Wastell, D; Broadhurst, K \& Hall, C (2010) When policy o'erleaps itself: The 'tragic tale' of the Integrated Children's System Critical Social Policy 30 (3) 405429 